\title{
On 2D 4-Finger Frictionless Optimal Grasps
}

\author{
Jordi Cornellà Raúl Suárez \\ Institut d'Organització i Control de Sistemes Industrials (IOC-UPC) \\ Av. Diagonal 647 Planta 11, 08028 Barcelona, SPAIN \\ Emails: jordi.cornella@upc.es, raul.suarez@upc.es
}

\begin{abstract}
The paper deals with the determination of optimal force-closure grasps for $2 \mathrm{D}$ polygonal objects. The problem is analyzed and some intrinsic properties of grasps are determined. The approach is applied to the determination of the position of a fourth finger given the positions of three other fingers. Moreover, the range of solutions that allow the force-closure property as well as the optimal value are analytically determined, checking only four points in the worst case. The algorithm has been implemented and numerical examples are included in the paper.
\end{abstract}

\section{INTRODUCTION}

Grasping and manipulation of objects using multi-finger mechanical hands has become a field of great interest in the two last decades. Good overviews of the state of the art in this field and related problems can be found in [14] and [2].

Grasps capable of resisting external disturbances satisfy one of the following properties: form-closure (the position of the fingers ensures the object immobility) or forceclosure (the forces applied by the fingers ensure the object immobility) [1]. Finding the optimal position of the fingers that produce a grasp satisfying either of these properties is a frequent problem. A criterion for grasp quality evaluation must be chosen, and several ones have therefore been proposed. Ferrari and Canny [6] proposed a criterion based on the maximum wrench that the grasp can safely resist, and algorithms for grasp synthesis based on this criterion were proposed by Pollard [13] and Borst et al. [3], though they do not generate optimal grasps. Ponce et al. [11] proposed the minimization of the distance between the object's center of mass and the geometric center of the grasping points. This criterion is used by Ding et al. [5], among others. One variation of the problem is the determination of independent regions on the object boundary such that a finger in each region ensures a form/force-closure grasp, thus dealing with some uncertainty in the finger positioning. The works of Ponce et al. [12] and Nguyen [10] are remarkable in this line.

In general, the algorithms proposed for the search of an optimal grasp are based on linear or non-linear programming, thereby implying an iterative procedure. This paper tackles the problem of finding optimal grasps

$$
0
$$

This work was partially supported by the CICYT projects DPI2001-2202 and DPI2002-03540 (in the sense of the most robust force-closure grasp) of 2D objects using the minimum number of frictionless contacts (four for 2D objects [9]) and the quality measure proposed by Ferrari and Canny, and avoiding iterative procedures. Some intrinsic properties of the problem that help in the solution search are presented here. In particular, the problem of determining the optimal position of a fourth finger given the positions of the other three and the contact edge for the fourth is solved in an analytical way (the problem has already been solved even for 3D objects [5], but the approach proposed here does not involve hard searches or iterative processes). Solving this problem is of special relevance in re-grasping actions. The main assumptions considered in this work are: 1) Grasped objects are planar and polygonal-shaped; 2) The object edge where the fourth finger will contact is given; 3) Forces applied by the fingers act only against the object boundary (positivity constraint); 4) Frictionless contacts (then the force-closure solution is also form-closure); 5) The fingertip is a point.

Note that there is no constraint regarding the number of fingers per edge. In this approach it is then possible to consider two fingers on the same edge (for polygonal objects a minimum of three edges must be contacted to allow a force-closure grasp).

\section{GRASP QUALITY MEASURE}

\section{A. In the Wrench Space}

One of the most intuitive and accepted criterion for quality evaluation of a grasp that satisfies the force-closure condition (hereafter called FC grasp) was proposed by Ferrari and Canny [6]. This criterion indicates the module of the maximum wrench that a grasp may resist regardless of the wrench direction: the larger the module, the better the grasp.

Each finger can exert a force $\boldsymbol{f}_{i}$ on the object boundary at each contact point (in the absence of friction, $\boldsymbol{f}_{i}$ is the applied force normal to the object boundary). Force $\boldsymbol{f}_{i}$ produces a torque $\boldsymbol{\tau}_{i}$ with respect to the object's center of mass, and the vector $\boldsymbol{\omega}_{i}=\left(\boldsymbol{f}_{i}^{T} \lambda \boldsymbol{\tau}_{i}^{T}\right)^{T}$ is called a wrench or a generalized force ( $\lambda$ defines the metric of the wrench space and, in order to have a physical meaning in terms of energy $\lambda$ is considered to be the radius of gyration. The proposed approach is valid for any value of $\lambda$; thus, for simplicity it is considered $\lambda=1$ ). 
The forces applied by the fingers can be subject to different constraints, depending on the characteristic of the grasp to be optimized. The constraint used in this work (other constraints with their physical and geometric meaning can be found in [9]) is that the total force exerted by all the fingers is limited, for instance, due to a maximum available power for all the finger actuators. This implies that the applied forces can generate a resultant wrench $\boldsymbol{\omega}=\sum_{i} \beta_{i} \boldsymbol{\omega}_{i}$ with $\sum_{i} \beta_{i} \leq 1$ and $\boldsymbol{\omega}_{i}$ being the wrench produced by forces satisfying $\left\|\boldsymbol{f}_{i}\right\|=1$. Geometrically, the resultant wrench can be any inside the polytope

$$
\mathcal{P}_{1}=\text { ConvexHull }\left(\bigcup_{i=1}^{n}\left\{\omega_{i}\right\}\right)
$$

Considering this constraint, the quality measure $Q$ of an FC grasp is given by the maximum wrench that the finger forces can generated in any direction of the wrench space:

$$
Q=\min _{\boldsymbol{\omega} \in \partial \mathcal{P}_{1}}\|\boldsymbol{\omega}\|
$$

where $\partial \mathcal{P}_{1}$ is the boundary of polytope $\mathcal{P}_{1}$.

In the case of $2 \mathrm{D}$ objects, $\mathcal{P}_{1}$ is a polyhedron and the quality measure is equivalent to the radius of the greatest sphere centered at the origin and fully contained in polyhedron $\mathcal{P}_{1}$.

The quality measure $Q$ is determined by one of the distances from the origin to the faces of $\mathcal{P}_{1}$. Consider three wrenches $\boldsymbol{\omega}_{i}(i=1, \ldots, 3)$ expressed in cylindrical coordinates as $\boldsymbol{\omega}_{i}=\left(\cos \alpha_{i}, \sin \alpha_{i}, \tau_{i}\right), \alpha_{i}$ being the angle that indicates the direction of $\boldsymbol{f}_{i}$. The distance $D$ from the origin to the face (plane) of $\mathcal{P}_{1}$ determined by $\boldsymbol{\omega}_{1}, \boldsymbol{\omega}_{2}$ and $\boldsymbol{\omega}_{3}$ can be written as a function of the torque of one of them; for instance, as a function of $\tau_{3}$ of $\boldsymbol{\omega}_{3}$, we obtain:

$$
D\left(\tau_{3}\right)=\left|\frac{k_{1}+k_{2} \tau_{3}}{\sqrt{\left(k_{3}+k_{4} \tau_{3}\right)^{2}+\left(k_{5}+k_{6} \tau_{3}\right)^{2}+k_{7}^{2}}}\right|
$$

where:

$$
\begin{aligned}
& k_{1}=\sin \left(\alpha_{3}-\alpha_{2}\right) \tau_{1}+\sin \left(\alpha_{1}-\alpha_{3}\right) \tau_{2} \\
& k_{2}=\sin \left(\alpha_{2}-\alpha_{1}\right) \\
& k_{3}=\left(\sin \alpha_{2}-\sin \alpha_{3}\right) \tau_{1}+\left(\sin \alpha_{3}-\sin \alpha_{1}\right) \tau_{2} \\
& k_{4}=\sin \alpha_{1}-\sin \alpha_{2} \\
& k_{5}=\left(\cos \alpha_{3}-\cos \alpha_{2}\right) \tau_{1}+\left(\cos \alpha_{1}-\cos \alpha_{3}\right) \tau_{2} \\
& k_{6}=\left(\cos \alpha_{2}-\cos \alpha_{1}\right) \\
& k_{7}=\sin \left(\alpha_{2}-\alpha_{3}\right)+\sin \left(\alpha_{3}-\alpha_{1}\right)+\sin \left(\alpha_{1}-\alpha_{2}\right)
\end{aligned}
$$

This function has four important properties (an example is shown in Fig. 1):

1. It is a continuous function (the denominator can only be zero if all the forces are in the same direction, and then the FC grasp is not possible).

2. It becomes zero at $\tau_{3}=-k_{1} / k_{2}$.

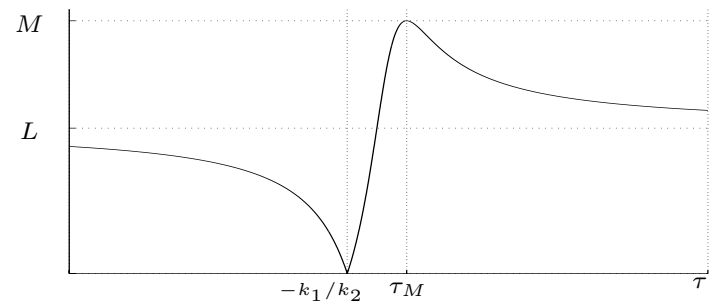

Fig. 1. Qualitative shape of distance $D(\tau)$

3. It tends to a finite positive value when $\tau_{3} \rightarrow \pm \infty$, $L=\lim _{\tau_{3} \rightarrow \pm \infty} D\left(\tau_{3}\right)=k_{2} / \sqrt{\left(k_{4}^{2}+k_{6}^{2}\right)}$.

4. It has only one maximum $M$ at

$$
\tau_{M}=\frac{\left(k_{3}^{2}+k_{7}^{2}+k_{5}^{2}\right) k_{2}-\left(k_{3} k_{4}+k_{5} k_{6}\right) k_{1}}{-k_{2}\left(k_{3} k_{4}+k_{5} k_{6}\right)+k_{1}\left(k_{4}^{2}+k_{6}^{2}\right)}
$$

\section{B. In the Force Space}

Similarly to Mirtich and Canny's application of the quality measure to two particular sets of forces and moments [7], the quality measure described in equation (2) can be applied in the force space (i.e. the 2D subspace of the wrench space defined by pure forces) as

$$
Q_{f}=\min _{\boldsymbol{f} \in \partial \mathcal{P}_{f}}\|\boldsymbol{f}\|
$$

where $\mathcal{P}_{f}$ is the polygon defined in the force space by the contact forces and $\partial \mathcal{P}_{f}$ is its boundary $\left(\mathcal{P}_{f}\right.$ coincides with the projection of $\mathcal{P}_{1}$ on the force space).

Proposition 1: Consider three wrenches that define a face of $\mathcal{P}_{1}$. If the component $\tau$ of one of them tends to $\pm \infty$, the distance between the origin of the wrench space and the face tends to the distance between the origin and the straight line defined by the projection of the other two wrenches on the force space.

Proof: The proof is straightforward from the property 3 of the distance in equation (3). If constants $k_{2}, k_{4}$ and $k_{6}$ are substituted by their expressions from equations (5),(7) and (9), then when $\tau_{3} \rightarrow \pm \infty$ the distance between the origin and $\overline{f_{1} f_{2}}$ is obtained.

Lemma 1: The quality measure in the force space, $Q_{f}$, is an Upper Bound for the grasp quality measure $Q$. $\diamond$

Proof: Consider a prism $\mathcal{P}_{1}^{\infty}$ in the wrench space with infinite edges parallel to the $\tau$-axis, such that the intersection of $\mathcal{P}_{1}^{\infty}$ with the force space is $\mathcal{P}_{f}$. The radius of the greatest sphere centered at the origin and inscribed in $\mathcal{P}_{1}^{\infty}$ is determined by the minimum distance from the origin to $\mathcal{P}_{f}$, i.e. $Q_{f}$.

Given the torques of the four contact forces, the corresponding wrenches generate a polyhedron $\mathcal{P}_{1}$ that satisfies $\mathcal{P}_{1} \subset \mathcal{P}_{1}^{\infty}$ by construction. Therefore, the greatest sphere centered at the origin and inscribed in $\mathcal{P}_{1}$ is smaller or equal to the one inscribed in $\mathcal{P}_{1}^{\infty}$. As a result, its maximum radius is $Q_{f}$. 


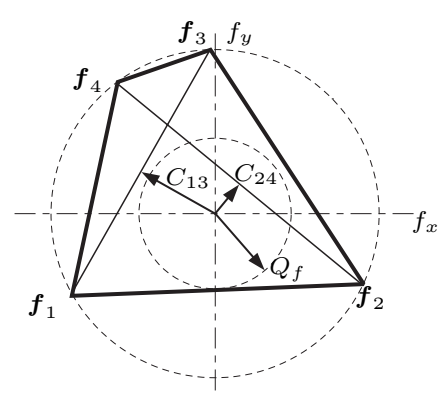

Fig. 2. Examples of the Upper Bound $Q_{f}$ and the Internal Bounds $C_{24}$ and $C_{13}$.

Definition 1: An Internal Bound, $C_{i j}$, of a grasp is the distance between the origin of the force space and a segment determined by two non-consecutive vertices $\boldsymbol{f}_{i}$ and $\boldsymbol{f}_{j}$ of $\mathcal{P}_{f}$.

Note that a four-finger grasp has two Internal Bounds. If the four applied forces have different directions, $\mathcal{P}_{f}$ is a quadrilateral polygon and the Internal Bounds are the distances from the origin to each diagonal of the polygon. Moreover, if there are two parallel applied forces (e.g. two fingers on the same object edge), $\mathcal{P}_{f}$ degenerates into a triangle and the Internal Bounds are given by the distances from the origin to two sides of the triangle. Fig. 2 shows an example of the Internal Bounds.

\section{OPTIMAL FOURTH CONTACT POINT}

Geometrically, the specific problem of determining the position of the fourth finger on the given edge is: given three points in the wrench space (i.e. $\boldsymbol{\omega}_{1}, \boldsymbol{\omega}_{2}, \boldsymbol{\omega}_{3}$ ) and the applied force $\boldsymbol{f}_{4}=\left(f_{x 4}, f_{y 4}\right)^{T}$, determine the component $\tau_{4}$ such that the polyhedron $\mathcal{P}_{1}$ defined by $\boldsymbol{\omega}_{1}, \boldsymbol{\omega}_{2}, \boldsymbol{\omega}_{3}$ and $\boldsymbol{\omega}_{4}=\left(f_{4 x}, f_{4 y}, \tau_{4}\right)^{T}$ includes the greatest possible sphere centered at the origin.

\section{A. Range of possible solutions}

In order to produce an FC grasp, $\mathcal{P}_{1}$ must contain the origin [8]. The following necessary and sufficient condition can be stated from this condition.

Necessary and Sufficient condition. Let $R_{f_{c_{4}}}$ be the range of $\tau_{4}$ that allows an FC grasp given $\boldsymbol{\omega}_{1}, \boldsymbol{\omega}_{2}, \boldsymbol{\omega}_{3}$ and $\boldsymbol{f}_{4}$, and let $\partial R_{f c_{4}}$ be the boundary of $R_{f c_{4}}$. Then, in order for a given $\tau_{4}^{*}$ to satisfy $\tau_{4}^{*} \in \partial R_{f c_{4}}$, it is necessary and sufficient that $0 \in \partial \mathcal{P}_{1}, \mathcal{P}_{1}$ being the polyhedron defined by $\boldsymbol{\omega}_{1}, \boldsymbol{\omega}_{2}, \boldsymbol{\omega}_{3}$ and $\boldsymbol{\omega}_{4}=\left(f_{4 x}, f_{4 y}, \tau_{4}^{*}\right)^{T}$.

Proof: It is straightforward since $\mathcal{P}_{1}$ is convex.

Using this condition, the extremes of $R_{f c_{4}}$ can be found as follows. First, three candidates are obtained from the intersection of the three planes defined by the sets of wrenches $\left\{\boldsymbol{\omega}_{2}, \boldsymbol{\omega}_{3}, 0\right\},\left\{\boldsymbol{\omega}_{1}, \boldsymbol{\omega}_{3}, 0\right\}$ and $\left\{\boldsymbol{\omega}_{1}, \boldsymbol{\omega}_{2}, 0\right\}$, respectively with the straight line determined by $x=f_{x 4}$, $y=f_{y 4}$. The three candidates are given by

$$
\tau_{4 j}=\frac{-A_{l i m j} f_{x 4}-B_{l i m j} f_{y 4}}{C_{l i m j}}
$$

where $A_{l i m j}, B_{l i m j}$ and $C_{l i m j}$ are the coefficients of each plane for $j=1, \ldots, 3$.

These candidates are then tested in the force-closure condition (i.e. the origin must be inside $\mathcal{P}_{1}$ ):

$$
\begin{array}{r}
\beta_{1 j}\left(f_{x 1}, f_{y 1}, \tau_{1}\right)^{T}+\beta_{2 j}\left(f_{x 2}, f_{y 2}, \tau_{2}\right)^{T}+ \\
\beta_{3 j}\left(f_{x 3}, f_{y 3}, \tau_{3}\right)^{T}=\left(f_{x 4}, f_{y 4}, \tau_{4 j}\right)^{T}
\end{array}
$$

where $\beta_{i j} \leq 0$ for $i=1, \ldots, 3$. Only one or two of the three candidates $\tau_{4 j}$ can be valid extremes of $R_{f c_{4}}$. Depending on the number of valid extreme points of $R_{f c_{4}}$, the type of range is:

Infinite range: if only one value $\tau_{4 j}$ satisfies the necessary and sufficient conditions (here denoted as $\tau_{4 k}$ ) then $R_{f c_{4}}$ is the range determined by $R_{f c_{4}}=\left[\tau_{4 k}, \infty\right)$ or $R_{f c_{4}}=\left(-\infty, \tau_{4 k}\right]$ such that $R_{f c_{4}}$ does not contain the other two $\tau_{4 j}$ with $j \neq k$.

Limited range: if two values $\tau_{4 j}$ satisfy the necessary and sufficient conditions (here denoted as $\tau_{4 k}$ and $\tau_{4 l}$ ) then $R_{f c_{4}}=\left[\tau_{4 k}, \tau_{4 l}\right]$.

Proposition 2: Given four wrenches $\boldsymbol{\omega}_{i}$ with $i=1, \ldots, 4$, the type of range $R_{f_{i}}$ of each $\tau_{i}$ is independent of the components $\tau_{i}$, and can be determined just from forces $\boldsymbol{f}_{i}$ (this is equivalent to saying that the type of range can be automatically determined knowing the edges of the object to be contacted by the fingers, regardless of the exact contact point).

Proof: Without loss of generality it will be proved that range $R_{f c_{4}}$ (i.e. for $\tau_{4}$ ) does not depend on components $\tau_{1}, \tau_{2}$ and $\tau_{3}$ of $\boldsymbol{\omega}_{1}, \boldsymbol{\omega}_{2}$ and $\boldsymbol{\omega}_{3}$, respectively.

The candidates to be extremes of $R_{f_{c_{4}}}$ are obtained from equation (13) as a function of only two other wrenches (consider, for instance $j=1$, i.e. candidate $\tau_{41}$ obtained from $\boldsymbol{\omega}_{2}$ and $\boldsymbol{\omega}_{3}$ ). This implies that one of the coefficients $\beta_{i 1}$ in equation (14) will be null when the sufficient condition is applied; for the case of candidate $\tau_{41}$, we obtain $\beta_{11}=0$, and equation (14) can be expressed as

$$
\begin{aligned}
\beta_{21} f_{x 2}+\beta_{31} f_{x 3} & =f_{x 4} \\
\beta_{21} f_{y 2}+\beta_{31} f_{y 3} & =f_{y 4} \\
\beta_{21} \tau_{2}+\beta_{31} \tau_{3} & =\tau_{41}
\end{aligned}
$$

i.e. three equalities with two unknowns, $\beta_{21}$ and $\beta_{31}$. Therefore, one of the equalities is linearly dependent on the other two. If $\boldsymbol{f}_{2}$ and $\boldsymbol{f}_{3}$ have different directions, (15) and (16) are independent, and equality (17) can be neglected. As a result, $\beta_{21}$ and $\beta_{31}$ can be determined as 
a function of $\boldsymbol{f}_{2}$ and $\boldsymbol{f}_{3}$ without taking into account $\tau_{2}$ and $\tau_{3}$ :

$$
\begin{aligned}
& \beta_{31}=\frac{\sin \left(\alpha_{4}-\alpha_{2}\right)}{\sin \left(\alpha_{3}-\alpha_{2}\right)} \\
& \beta_{21}=\frac{\cos \left(\alpha_{4}\right) \sin \left(\alpha_{3}-\alpha_{2}\right)-\cos \left(\alpha_{3}\right) \sin \left(\alpha_{4}-\alpha_{2}\right)}{\cos \left(\alpha_{2}\right) \sin \left(\alpha_{3}-\alpha_{2}\right)}
\end{aligned}
$$

where $\alpha_{i}$ is the angle that indicates the direction of $\boldsymbol{f}_{i}$ in the force space. According to equation (14), if $\beta_{21} \leq 0$ and $\beta_{31} \leq 0$, then $\tau_{41}$ is an extreme of $R_{f c_{4}}$ (even when the exact value of $\tau_{41}$ is not known!). In the same way, $\beta_{12}$ and $\beta_{32}$ are obtained for $\tau_{42}$, and $\beta_{13}$ and $\beta_{23}$ for $\tau_{43}$, and the analysis of their signs determines if $\tau_{42}$ and $\tau_{43}$ are extremes of $R_{f c_{4}}$.

If either of the applied forces has the same direction (e.g. two fingers on the same edge), equations (15) and (16) have no solution and the corresponding candidate does not exist. Then, since at least two of the three applied forces have different directions, two candidates are possible.

Then, although $\tau_{41}, \tau_{42}$ and $\tau_{43}$ are not known, it is possible to know how many of them are extremes of $R_{f c_{4}}$ and therefore the corresponding type of range.

Equivalent reasoning can be applied for $R_{f c_{1}}, R_{f c_{2}}$ and $R_{f c_{3}}$.

\section{B. Determination of the Optimal Value}

Proposition 3: Given three wrenches, $\boldsymbol{\omega}_{i}, \boldsymbol{\omega}_{j}$ and $\boldsymbol{\omega}_{k}$ (with $\{i, j, k\}=\{1,2,3\}$ ) and $\boldsymbol{f}_{4}$, the value $\tau_{4}$ that produces an optimal grasp $\left(\tau_{4 o p t}\right)$ can be analytically determined according to the following four cases. Let $C_{i j}$ be the Internal Bound that does not depend on $\boldsymbol{f}_{4}$ and $D_{\rho}$ the distance from the origin to the plane defined by $\boldsymbol{\omega}_{\nu}$, $\boldsymbol{\omega}_{\sigma}$, and $\boldsymbol{\omega}_{4}$, with $\{\rho, \nu, \sigma\}=\{i, j, k\}$. Then,

Case 1: $R_{f c_{4}}$ is Infinite and $C_{i j} \geq Q_{f}$; then,

$\tau_{4 o p t}= \pm \infty$ according to $R_{f c_{4}}$.

Case 2: $R_{f c_{4}}$ is Limited and $C_{i j} \geq Q_{f}$; then, there are three candidates for $\tau_{4 o p t}$ given by the solutions of

$$
\begin{aligned}
& D_{i}\left(\tau_{4}\right)=D_{j}\left(\tau_{4}\right) \\
& D_{i}\left(\tau_{4}\right)=D_{k}\left(\tau_{4}\right) \\
& D_{j}\left(\tau_{4}\right)=D_{k}\left(\tau_{4}\right)
\end{aligned}
$$

Case 3: $R_{f_{c_{4}}}$ is Limited and $C_{i j}<Q_{f}$; then, there are four candidates for $\tau_{4 o p t}$ given by the three obtained in Case 2 plus the value of $\tau_{4}$ that maximizes $D_{k}$, i.e. the solution of

$$
\frac{\partial D_{k}\left(\tau_{4}\right)}{\partial \tau_{4}}=0
$$

If more than one of these candidates belongs to $R_{f c_{4}}$, they must be tested to identify $\tau_{4 o p t}$.
Case 4: $R_{f c_{4}}$ is Infinite and $C_{i j}<Q_{f}$; then, there are three candidates for $\tau_{4 o p t}$ given by the solutions of

$$
\begin{aligned}
D_{k}\left(\tau_{4}\right) & =D_{i}\left(\tau_{4}\right) \\
D_{k}\left(\tau_{4}\right) & =D_{j}\left(\tau_{4}\right) \\
\frac{\partial D_{k}\left(\tau_{4}\right)}{\partial \tau_{4}} & =0
\end{aligned}
$$

If none of the candidates belong to $R_{f c_{4}}$, then $\tau_{4 o p t}= \pm \infty$ according to $R_{f c_{4}}$.

Proof: The proofs of the four cases are based on

- $Q_{f}>Q\left(\tau_{4}\right) \forall \tau_{4} \in R_{f c_{4}}$.

- $L_{\rho}=\lim _{\tau_{4} \rightarrow \pm \infty} D_{\rho}\left(\tau_{4}\right)$ is finite $(\rho=i, j, k)$.

- $D_{\rho}\left(\tau_{4}\right)$ has only one maximum $M_{\rho}(\rho=i, j, k)$.

- $Q\left(\tau_{4}\right)$ is defined by pieces of $D_{\rho}\left(\tau_{4}\right)(\rho=i, j, k)$.

Case 1: $R_{f c_{4}}$ Infinite, $C_{i j} \geq Q_{f}$

1. If $C_{i j} \geq Q_{f} \Rightarrow \forall \rho L_{\rho} \geq Q_{f}, \rho=i, j, k$.

2. $D_{\rho}\left(\tau_{4}\right)$ has only one maximum $M_{\rho}$.

3. From 1 and 2, we obtain $M_{\rho}>Q_{f}$.

4. From 3, $Q\left(\tau_{4}\right)<M_{\rho}$; then from 2 , all the pieces of $D_{\rho}\left(\tau_{4}\right)$ that define $Q\left(\tau_{4}\right)$ are monotonous.

5. If $R_{f c_{4}}$ is Infinite, there is only one $\tau_{4}{ }^{*}$ where $Q\left(\tau_{4}{ }^{*}\right)=0$; then from 4 , the pieces of $D_{\rho}\left(\tau_{4}\right)$ belonging to $Q\left(\tau_{4}\right)$ increase if $R_{f c_{4}}=\left[\tau_{4}{ }^{*}, \infty\right)$ or decrease if $R_{f c_{4}}=\left(-\infty, \tau_{4}{ }^{*}\right]$. Then, $\tau_{4 o p t}= \pm \infty$ according $R_{f c_{4}}$.

Case 2: $R_{f c_{4}}$ Limited, $C_{i j} \geq Q_{f}$

1. Same as steps 1 to 4 from Case 1 ; then, all the pieces of $D_{\rho}\left(\tau_{4}\right)$ that define $Q\left(\tau_{4}\right)$ are monotonous.

2. If $R_{f_{c_{4}}}$ is Limited, there are two values $\tau_{4}^{*}$ and $\tau_{4}^{*}$ where $Q\left(\tau_{4}^{*}\right)=Q\left(\tau_{4}^{*}\right)=0$; then, there are increasing and decreasing pieces in $Q\left(\tau_{4}\right)$.

3. From 1 and 2 , the optimal of $Q\left(\tau_{4}\right)$ is located where an increasing piece and a decreasing piece intersect each other.

4. The values of $\tau_{4}$ where two pieces of $Q\left(\tau_{4}\right)$ intersect each other are the solutions of equations (20) to (22) and $\tau_{4 o p t}$ is one of these solutions.

Case 3: $R_{f c_{4}}$ Limited, $C_{i j}<Q_{f}$

1. If $C_{i j} \leq Q_{f} \Rightarrow L_{\rho} \geq Q_{f}$ with $\rho=i, j$.

2. Same as steps 1 to 4 from Case 1 but considering only values $\rho=i, j$. Then, all the pieces of $D_{\rho}\left(\tau_{4}\right)$ that define $Q\left(\tau_{4}\right)$ are monotonous while the pieces of $Q\left(\tau_{4}\right)$ from $D_{k}\left(\tau_{4}\right)$ might not be.

3. Same as step 2 from Case 2.

4. From 2 and 3, the optimal of $Q\left(\tau_{4}\right)$ is located where an increasing piece and a decreasing piece intersect each other, or where $D_{k}\left(\tau_{4}\right)$ has the maximum $M_{k}$ (i.e. $D_{k}\left(\tau_{4}\right)$ changes from increasing to decreasing).

5. The values of $\tau_{4}$ where two pieces of $Q\left(\tau_{4}\right)$ intersect each other are the solutions of equations (20) to (22), the value of $\tau_{4}$ where $M_{k}$ takes place is the solution of equation (23) and $\tau_{4 o p t}$ is one of these solutions. 
Case 4: $R_{f c_{4}}$ Infinite, $C_{i j}<Q_{f}$

1. Same as steps 1 and 2 from Case 3. Then, all the pieces of $D_{\rho}\left(\tau_{4}\right)$ that define $Q\left(\tau_{4}\right)$ are monotonous for $\rho=i, j$ while the pieces of $Q\left(\tau_{4}\right)$ from $D_{k}\left(\tau_{4}\right)$ might not be.

2. If $R_{f c_{4}}$ is Infinite, there is only one $\tau_{4}{ }^{*}$ where $Q\left(\tau_{4}^{*}\right)=0$.

3. From 1 and 2 , the pieces of $Q\left(\tau_{4}\right)$ from $D_{\rho}\left(\tau_{4}\right)$ increase if $R_{f c_{4}}=\left[\tau_{4}{ }^{*}, \infty\right)$ or decrease if $R_{f c_{4}}=\left(-\infty, \tau_{4}^{*}\right]$, while the pieces of $Q\left(\tau_{4}\right)$ from $D_{k}\left(\tau_{4}\right)$ might not be.

4. From 3, any transition from an increasing piece of $Q\left(\tau_{4}\right)$ to a decreasing one involves $D_{k}\left(\tau_{4}\right)$.

5. The values of $\tau_{4}$ where $Q\left(\tau_{4}\right)$ changes from increasing to decreasing are the solutions of equations (24) to (26) and $\tau_{4 o p t}$ is one of these solutions.

\section{Constraint of the edge length}

It is possible that the optimal value of $\tau_{4}$ found above cannot be generated due to the finite length of the contact edges. This constraint is included here.

The length of the edge for the fourth finger defines a new range, $R_{4}$, of values of $\tau_{4}$ that are physically possible in the real execution of the grasping. Considering $R_{4}$, $R_{f c_{4}}$ and $\tau_{4 o p t}$, three cases are possible:

$R_{4} \cap R_{f c_{4}}=\varnothing$ : An FC grasp is not possible by placing the fourth finger on the assigned edge.

$R_{4} \cap R_{f c_{4}} \neq \varnothing$ and $\tau_{4 o p t} \in\left(R_{4} \cap R_{f c_{4}}\right)$ : The actual optimal position is that corresponding to $\tau_{4 o p t}$.

$R_{4} \cap R_{f c_{4}} \neq \varnothing$ and $\tau_{4 o p t} \notin\left(R_{4} \cap R_{f c_{4}}\right)$ : The actual optimal position is the extreme of $\left(R_{4} \cap R_{f c_{4}}\right)$ closer to $\tau_{4 o p t}$, because function $Q\left(\tau_{4}\right)$ grows monotonously towards $Q\left(\tau_{4 o p t}\right)$ inside $R_{f c_{4}}$.

Note that the optimal position may be on an object vertex or close to it. In this case, it is necessary to consider a security distance.

\section{Efficient Procedure}

When Proposition 3 is applied, with the exception of Case 1 where $\tau_{4 o p t}$ can be directly found, it is necessary to solve three four-order equations. If these equations are solved in the right sequence, $\tau_{4 o p t}$ may be obtained without arriving to solve all of them. The right sequence is determined by the following steps:

1. Check that $R_{4} \cap R_{f_{4}} \neq \varnothing$.

2. Find $\tau_{4}^{\prime}$ from $D_{j}\left(\tau_{4}\right)=D_{k}\left(\tau_{4}\right), j$ and $k$ being such that:

Cases 2 and 3: $D_{j}\left(\tau_{4}\right)$ and $D_{k}\left(\tau_{4}\right)$ make $Q\left(\tau_{4}\right)=0$.

Case 4: $D_{j}\left(\tau_{4}\right)$ makes $Q\left(\tau_{4}\right)=0$ and $D_{k}\left(\tau_{4}\right) \rightarrow C_{i j}$ when $\tau_{4} \rightarrow \pm \infty$.

3. Evaluate $D_{\rho}\left(\tau_{4}{ }^{\prime}\right), \rho=i, j, k$. Two situations are possible: a. If $D_{i}\left(\tau_{4}{ }^{\prime}\right) \geq D_{j}\left(\tau_{4}{ }^{\prime}\right)=D_{k}\left(\tau_{4}{ }^{\prime}\right)$ :

it is not necessary to solve $D_{i}\left(\tau_{4}\right)=D_{j}\left(\tau_{4}\right)$ and

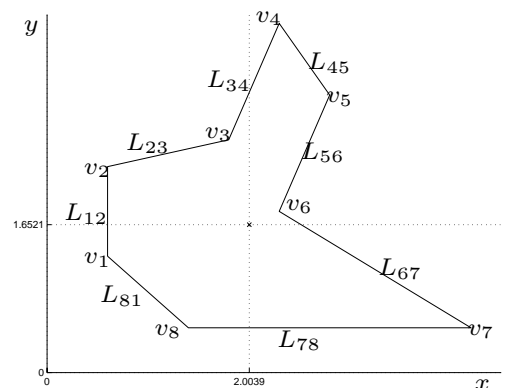

\begin{tabular}{c|c|c} 
& $x$ & $y$ \\
\hline$v_{1}$ & 0.6 & 1.3 \\
\hline$v_{2}$ & 0.6 & 2.3 \\
\hline$v_{3}$ & 1.8 & 2.6 \\
\hline$v_{4}$ & 2.3 & 3.9 \\
\hline$v_{5}$ & 2.8 & 3.1 \\
\hline$v_{6}$ & 2.3 & 1.8 \\
\hline$v_{7}$ & 4.2 & 0.5 \\
\hline$v_{8}$ & 1.4 & 0.5
\end{tabular}

Fig. 3. $2 D$ object and coordinates of its vertices

$D_{i}\left(\tau_{4}\right)=D_{k}\left(\tau_{4}\right)$ because the solutions of these equations never belong to $Q\left(\tau_{4}\right)$; then, $\tau_{4}{ }^{\prime}$ is a candidate to be $\tau_{4 o p t}$.

b. If $D_{i}\left(\tau_{4}{ }^{\prime}\right)<D_{j}\left(\tau_{4}{ }^{\prime}\right)=D_{k}\left(\tau_{4}{ }^{\prime}\right) \Rightarrow \tau_{4 o p t} \neq \tau_{4}{ }^{\prime}$ :

it is necessary to find $\tau_{4}{ }^{\prime \prime}$ from $D_{i}\left(\tau_{4}\right)=D_{j}\left(\tau_{4}\right)$ and $\tau_{4}{ }^{\prime \prime \prime}$ from $D_{i}\left(\tau_{4}\right)=D_{k}\left(\tau_{4}\right)$; then, $\tau_{4}{ }^{\prime \prime}$ and $\tau_{4}{ }^{\prime \prime \prime}$ are candidates to be $\tau_{4 o p t}$.

4. For Cases 3 and 4: Find $\tau_{4}{ }^{\prime \prime \prime \prime}$ that maximizes $D_{k}\left(\tau_{4}\right)$; then, $\tau_{4}{ }^{\prime \prime \prime \prime}$ is a candidate to be $\tau_{4 o p t}$.

5. Compute $D_{\rho}\left(\tau_{4}\right), \rho=i, j, k$, for the corresponding candidates, and select as $\tau_{4 o p t}$ the candidate that produces the maximum minimum.

\section{EXAMPLES}

In this section, some numerical examples of the proposed methodology are presented. A detailed example of each case can be found in [4]. Fig. 3 shows the object used in the examples. For each finger $i$ we have: the edge that will contact, the direction $\alpha_{i}$ of the applied force (orthogonal to the edge) and the range $R_{i}=\left[\tau_{\text {min }_{i}}, \tau_{\text {max }_{i}}\right]$ of possible actual torques produced on the contact edge.

Example of Case 1. The initial data are:

\begin{tabular}{c|c|c|c|c|c} 
finger & edge & $\alpha_{i}$ & $\tau_{\text {min }_{i}}$ & $\tau_{\text {max }_{i}}$ & $\tau_{i}$ \\
\hline 1 & $L_{12}$ & 0 & -0.6479 & 0.3521 & -0.2 \\
\hline 2 & $L_{23}$ & 4.9574 & 0.0321 & 1.2048 & 0.5 \\
\hline 3 & $L_{56}$ & 2.7744 & 0.2443 & 1.6372 & 1 \\
\hline 4 & $L_{67}$ & 4.1123 & -2.4630 & -0.1609 & $\tau_{4 o p t} ?$
\end{tabular}

Procedure:

1. $R_{f c_{4}}=(-\infty,-1.7568] \Rightarrow R_{4} \cap R_{f c_{4}} \neq \varnothing$

2. $Q_{f}=0.1826$ (determined by $\overline{f_{1} f_{3}}$ )

3. $C_{23}=0.4612$ (determined by $\overline{f_{2} f_{3}}$ )

4. $C_{23}>Q_{f}, R_{f_{c_{4}}}$ is Infinite $\Rightarrow$ Case 1

5. Then, $\tau_{4 o p t}=-\infty \notin R_{4} \cap R_{f_{4}}$

Solution: $\tau_{4 o p t}=-2.4630$ (extreme of $L_{67}$ ),

$$
Q=D_{2}\left(\tau_{4 o p t}\right)=0.0383 \text {. }
$$

The distances to the unknown faces of $\mathcal{P}_{1}$ as a function of $\tau_{4}$ and the obtained solution are shown in Fig. 4. The optimal solution is an extreme of an edge and it is so indicated; in the real grasp, a given security distance from the edge vertex should be considered. 


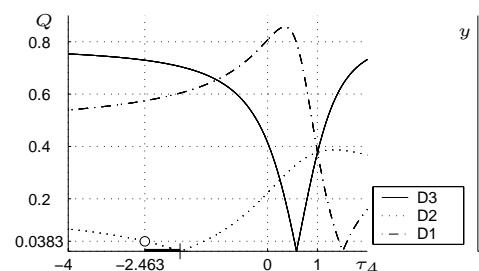

a)

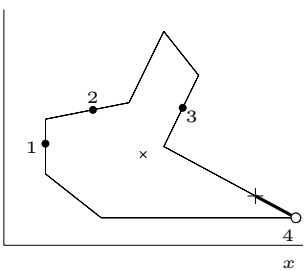

b)
Fig. 4. Example of Case 1: a) Distances to the faces of $\mathcal{P}_{1}$, range of possible solutions $R_{4} \cap R_{f_{4}}$ (dark segment) and maximum $Q$ (white circle); b) possible solutions on the object edge (dark segment) and optimal position for the fourth finger (white circle).

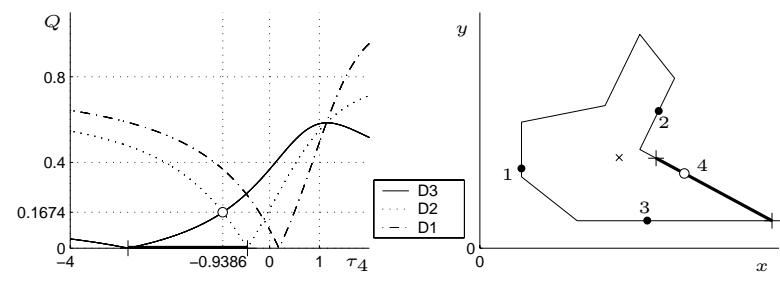

b)

Fig. 5. Example of Case 3: a) Distances to the faces of $\mathcal{P}_{1}$, range of possible solutions $R_{4} \cap R_{f_{4}}$ (dark segment) and maximum $Q$ (white circle); b) possible solutions on the object edge (dark segment) and optimal position for the fourth finger (white circle).

Example of Case 3. The initial data are:

\begin{tabular}{c|c|c|c|c|c} 
finger & edge & $\alpha_{i}$ & $\tau_{\text {min }_{i}}$ & $\tau_{\max _{i}}$ & $\tau_{i}$ \\
\hline 1 & $L_{12}$ & 0 & -0.6479 & 0.3521 & 0.2 \\
\hline 2 & $L_{56}$ & 2.7744 & 0.2443 & 1.6372 & 1 \\
\hline 3 & $L_{78}$ & 1.5708 & -0.6039 & 2.1961 & 0.4 \\
\hline 4 & $L_{67}$ & 4.1123 & -2.4630 & -0.1609 & $\tau_{4 o p t} ?$
\end{tabular}

Procedure:

1. $R_{f c_{4}}=[-2.8409,-0.4430] \Rightarrow R_{4} \cap R_{f c_{4}} \neq \varnothing$

2. $Q_{f}=0.4665$ (determined by $\overline{f_{1} f_{4}}$ )

3. $C_{12}=0.1826$ (determined by $\overline{f_{1} f_{2}}$ )

4. $C_{12}<Q_{f}, R_{f_{c_{4}}}$ is Limited $\Rightarrow$ Case 3 .

5. From $D_{3}=D_{2} \Rightarrow \tau_{4}{ }^{\prime}=-0.9386 \in R_{f_{4}} \cap R_{4}$ then $D_{1}\left(\tau_{4}{ }^{\prime}\right)=0.3686, D_{2}\left(\tau_{4}{ }^{\prime}\right)=D_{3}\left(\tau_{4}{ }^{\prime}\right)=0.1674$

7. $D_{1}\left(\tau_{4}{ }^{\prime}\right) \geq D_{3}\left(\tau_{4}{ }^{\prime}\right)=D_{2}\left(\tau_{4}{ }^{\prime}\right)$

8. From $\partial D_{3} / \partial \tau_{4}=0 \Rightarrow \tau_{4}{ }^{\prime \prime \prime \prime}=1.1537 \notin R_{f c_{4}}$

Solution: $\tau_{4 o p t}=-0.9386, Q=0.1674$

The distances to the unknown faces of $\mathcal{P}_{1}$ as a function of $\tau_{4}$ and the obtained solution are shown in Fig. 5.

\section{CONCLUSIONS}

The paper deals with the problem of determining optimal grasps of 2D objects using four fingers. An approach to the deterministic solution of this problem is proposed using the quality measure introduced by Ferrari and Canny [6] and considering the particular case where three fingers are already positioned and the position of the fourth must be determined. The extension to the general case of four unknown finger positions is under development. The force space is used to establish bounds for the quality measure that are quite useful for determining the optimal grasp and proving some intrinsic properties of the problem, like the type of range of solution on each object edge. Although this property is marginally used in the particular case of looking for the position of the fourth finger, it seems quite a significant intrinsic property for the solution search in the general case of four unknown finger positions.

\section{REFERENCES}

[1] Bicchi, A. On the Closure Properties of Robotics Grasping. Int. J. Robot. Res., 14(4) pp. 319-334, 1995.

[2] Bicchi, A. Hands for Dexterous Manipulation and Robust Grasping: A Difficult Road Toward Simplicity. IEEE Trans. Robot. and Aut., 16(6) pp.652-662, 2000.

[3] Borst, Ch., Fischer, M. and Hirzinger, G. A Fast and Robust Grasp Planner for Arbitrary 3D Objects. In Proc. IEEE ICRA, pp. 1890-1896. Detroit, 1999.

[4] Cornellà, J. and Suárez, R. On 2D 4-finger frictionless optimal grasps. Tech. Rep. IOC-DT-P-2003-03.

[5] Ding, D., Liu, Y.-H. and Wang, S. Computing 3-D Optimal Form-Cosure Grasps. In Proc. IEEE ICRA, pp.3573-3578. San Francisco, 2000.

[6] Ferrari, C. and Canny, J. Planning Optimal Grasps. In Proc. IEEE ICRA, pp.2290-2295. Nice, 1992.

[7] Mirtich, B. and Canny, J. Easily Computable Optimum Grasps in 2-D and 3-D. In Proc. IEEE ICRA, pp.739-747, San Diego, 1994.

[8] Mishra, B., Schwartz, J.T. and Sharir. M. On the Existence and Synthesis of Multifinger Positive Grips. Algorithmica, Special Issue: Robotics, 2(4) pp.541558, 1987.

[9] Mishra, B. Grasp Metrics: Optimality and Complexity. Algorithmic Foundations of Robotics, pp.137166, A.K. Peters, Wellesley, MA, 1995.

[10] Nguyen, V.D. Constructing Force-Closure Grasps. Int. J. Robot. Res., 7(3) pp.3-16, 1988.

[11] Ponce, J. and Faverjon, B. On Computing Three Finger Force-Closure Grasp of Polygonal Objects. IEEE Trans. Robot. and Aut., 11(6) pp.868-881, 1995.

[12] Ponce, J., Sullivan, S, Sudsang, A., Boissonnat, J.D. and Merlet, J.-P. On Computing Four-Finger Equilibrium and Force-Closure Grasps of Polyhedral Objects. Int. J. Robot. Res., 16, (1), pp. 11-35, 1997.

[13] Pollard, N. Synthesizing Grasps from Generalized Prototypes. In proc. IEEE ICRA, pp.2124-2130. Minneapolis, 1996.

[14] Shimoga, K.B. Robot Grasp Synthesis: A Survey. Int. J. Robot. Res., 15(3) pp.230-266, 1996. 